\title{
Verificação da eficiência do modelo de Mohler na resposta do comportamento de vigas mistas de madeira e concreto
}

\author{
Verification of the efficiency of Mohler model in the \\ response of the behavior of wood and concrete composite \\ beams
}

\section{Julio Cesar Molina \\ Marcia Almeida de Amaral Arcos da Silva Raimundo Pereira de Vasconcelos}

Julio Cesar Molina Universidade Estadual Paulista Júlio de Mesquita Filho Itapeva - SP - Brasil

Marcia Almeida de Amaral Arcos Universidade Federal do Amazonas Manaus - AM - Brasil

Raimundo Pereira de Vasconcelos Universidade Federal do Amazonas Manaus - AM - Brasil

Recebido em 23/01/14 Aceito em 25/12/14

\section{Resumo}

$\mathbf{N}$

este trabalho de pesquisa avaliou-se o comportamento estrutural do sistema misto madeira e concreto para tabuleiros de pontes com ênfase nos conectores metálicos de cisalhamento. Os ensaios experimentais foram realizados em corpos de prova mistos e em vigas mistas de madeira e concreto com sistema de conexão metálica, disposto em X, a partir da utilização de parafusos do tipo CS100900. Todos os corpos de prova e vigas foram submetidos a solicitações estáticas com carregamentos aplicados até a ruptura, considerando-se os diversos materiais envolvidos na ligação mista para a obtenção da resistência e rigidez do sistema de conexão. Os resultados experimentais de rigidez das vigas foram comparados com os resultados analíticos fornecidos pelo modelo de Mohler, tendo apresentado boa concordância para carregamentos de serviço. A partir dos resultados experimentais obtidos constatou-se que os danos mais significativos ocorreram nas regiões dos conectores.

Palavras-chaves: Vigas mistas de madeira e concreto. Modelo de Mohler. Conectores de cisalhamento.

\section{Abstract}

The aim of this research study was to evaluate the structural behaviour of the wood and concrete composite system for bridge decks with emphasis on the metal shear connectors. Experimental tests were performed on composite specimens and wood and concrete beams with a metallic connector system in an X position, using CS100900-type screws. All specimens and beams were submitted to static loads until failure in order to obtain the strength and stiffness of the connection system. The experimental results for the stiffness of the beams were compared with the analytical results obtained through the Mohler model, presenting good equivalence for service loads. The experimental results obtained demonstrate that the most significant damage in composite systems occurred in the connectors' areas.

Keywords: Wood concrete composite beams. Mohler model. Shear connectors. 


\section{Introdução}

Atualmente, ao se conceber um projeto de engenharia, procura-se aproveitar as melhores propriedades dos materiais, a fim de minimizar o custo e o desperdício. A maioria das pontes de madeira no Brasil encontra-se deteriorada e, em muitos casos, imprópria para utilização. Isso se deve à má utilização da madeira como material estrutural e também à falta de conhecimento de suas propriedades e possibilidades de aplicação. A maioria dos problemas nas pontes de madeira é referente à soltura das pranchas, ao desgaste superficial e ao apodrecimento por falta de tratamento preservante. Uma alternativa para solucionar esses problemas é a utilização de tabuleiros mistos de madeira e concreto com sistema de conexão metálico. Assim, no aspecto estrutural, pode-se aumentar a resistência e a rigidez da estrutura como um todo e, no aspecto ambiental, pode-se utilizar de maneira mais racional cada um dos materiais de construção envolvidos no sistema misto. Nesse contexto, busca-se utilizar as melhores propriedades dos materiais, ou seja, a capacidade resistente do concreto à compressão, e a da madeira, à tração. Além disso, a utilização do concreto em conjunto com a madeira proporciona para a madeira maior proteção contra o desgaste por abrasão, além de diminuir a vibração do tabuleiro em função da passagem dos veículos. No entanto, segundo Branco e Cruz (2003), é necessária a utilização de um sistema de conexão (ligação) entre os materiais para que ambos trabalhem em conjunto. Este trabalho de pesquisa apresenta os resultados experimentais de resistência e rigidez do sistema de conexão metálico formado por parafusos do tipo CS100900, dispostos em X, em corpos de provas mistos e em vigas mistas de madeira e concreto. Os resultados apresentados são comparados com os resultados teóricos de rigidez fornecidos pelo modelo de Mohler e poderão contribuir para o dimensionamento de tabuleiros mistos de pontes, já que atualmente a bibliografia relacionada ao assunto encontra-se em fase de desenvolvimento no Brasil. Os tabuleiros mistos de madeira e concreto apresentam-se como uma ótima alternativa para resolver a maioria dos problemas apresentados nas pontes de madeira no país.

\section{Modelo de Mohler}

Um dos modelos de cálculo mais utilizados no dimensionamento de tabuleiros mistos é o modelo de viga equivalente, proposto por Mohler, que está apresentado na norma europeia Eurocode 5 (EUROPEAN..., 2001). Esse modelo foi adaptado para estruturas mistas de madeira e concreto, e considera o tabuleiro misto como uma viga equivalente, de seção transversal $\mathrm{T}$, formada por uma laje armada de concreto, unida a uma viga de madeira, através de um sistema de conexão metálico, como mostra a Figura 1. No caso dos tabuleiros mistos de madeira e concreto, a largura $\mathrm{b}_{\mathrm{c}}$ da mesa da viga pode ser considerada como a distância de centro a centro entre vigas de madeira, tendo em vista a proximidade entre elas.

\section{Figura 1 - Seção transversal da viga equivalente e respectivas tensões internas na seção mista}

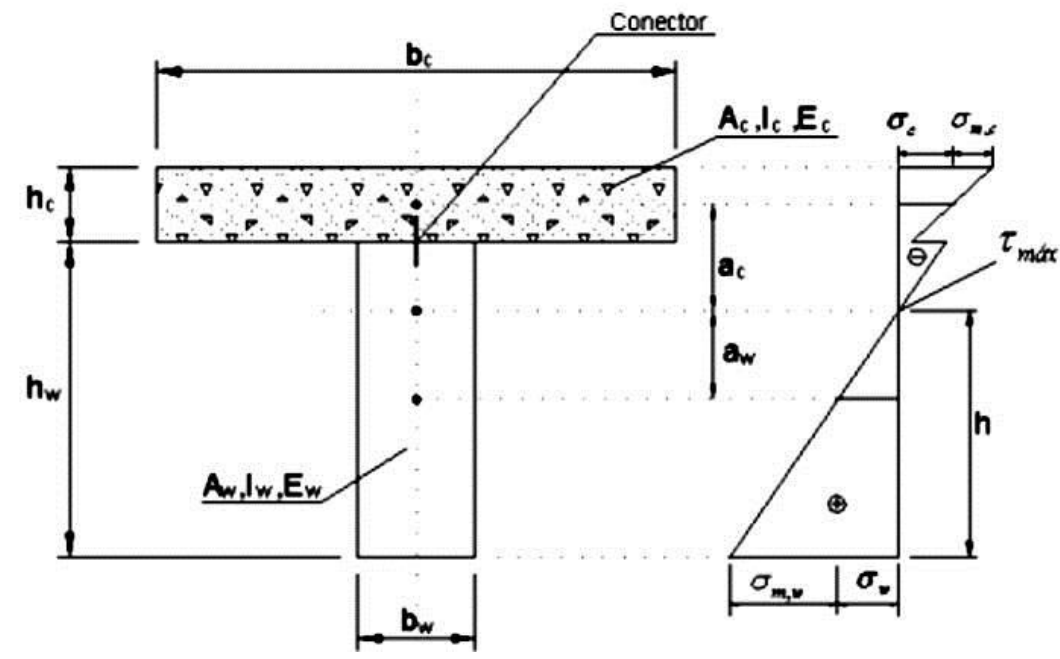

Fonte: modificado de Molina (2008). 
Neste modelo, a partir do módulo de deslizamento do conector utilizado, define-se o fator de redução de inércia do conjunto. A redução é feita para o material que apresentar o maior módulo de elasticidade (Eq. 1 e 2).

$\gamma_{\mathrm{w}}=1$

Eq. 1

$\gamma_{c}=\left[1+\frac{\pi^{2} \cdot E_{c} \cdot A_{c} \cdot S}{K \cdot L^{2}}\right]^{-1}$

Eq. 2

Onde:

$\mathrm{E}_{\mathrm{c}}=$ módulo de elasticidade do concreto na compressão;

$\mathrm{A}_{\mathrm{c}}=$ área da seção transversal da peça do concreto; $\mathrm{s}=$ espaçamento entre os conectores;

$\mathrm{K}$ = módulo de deslizamento do conector utilizado;

$\mathrm{L}=$ vão livre considerado para a viga mista.

Vale mencionar que a maneira mais eficiente de se conhecer o verdadeiro comportamento do conector é através do módulo de deslizamento $\mathrm{K}$ dos conectores, que é obtido pelo ensaio de cisalhamento direto realizado em corpos de prova. O Eurocode 5 (EUROPEAN..., 2001) apresenta equações para a determinação do módulo de deslizamento para conectores verticais e não apresenta equações para conectores dispostos em $\mathrm{X}$. As distâncias entre os centros de gravidade dos materiais na seção mista até a linha neutra (LN) da peça são dadas por (Eq. 3 e 4$)$ :

$$
\begin{aligned}
& a_{w}=\frac{\gamma_{c} \cdot E_{c} \cdot A_{c} \cdot\left(h_{c}+h_{w}\right)}{2 \cdot\left(\gamma_{c} \cdot E_{c} \cdot A_{c}+\gamma_{w} \cdot E_{w} \cdot A_{w}\right)} \\
& a_{c}=\left[\frac{h_{c}+h_{w}}{2}\right]-a_{w}
\end{aligned}
$$

Onde:

$\mathrm{a}_{\mathrm{c}}=$ distância do centroide da área de concreto até a linha neutra;

$\mathrm{a}_{\mathrm{w}}=$ distância do centroide da área de madeira até a linha neutra;

$\mathrm{h}_{\mathrm{c}}=$ altura da laje de concreto; $\mathrm{e}$

$\mathrm{h}_{\mathrm{w}}=$ altura da viga de madeira.

A influência do deslizamento na ligação composta é considerada mediante o seguinte produto de rigidez efetivo (Eq. 5):

$$
\left(E I_{e f}\right)=E_{c} \cdot I_{c}+\gamma_{c} \cdot E_{c} \cdot A_{c} \cdot a_{c}^{2}+E_{w} \cdot I_{w}+\gamma_{w} \cdot E_{w} \cdot A_{w} \cdot a_{w}^{2}
$$

Onde:

$I_{c}=$ momento de inércia da seção de concreto; $\mathrm{e}$
$\mathrm{I}_{\mathrm{w}}=$ momento de inércia da seção de madeira.

Sendo (Eq. 6 e 7):

$\mathrm{I}_{\mathrm{c}}=\frac{\mathrm{b}_{\mathrm{c}} \cdot \mathrm{h}_{\mathrm{c}}^{3}}{12}$

Eq. 6

$\mathrm{I}_{\mathrm{w}}=\frac{\mathrm{b}_{\mathrm{w}} \cdot \mathrm{h}_{\mathrm{w}}^{3}}{12}$

$\mathrm{Na}$ seção mista, a verificações de resistência a serem efetuadas são referentes aos estados limites últimos (tensões internas: normais e cisalhantes) e também aos estados limites de utilização ou de serviço (ligação e deslocamentos). A verificação da tensão normal de compressão na mesa de concreto, neste caso, é efetuada por (Eq. 8 a 10):

$$
\begin{aligned}
& \sigma_{c}=\gamma_{c} \cdot E_{c} \cdot a_{c} \cdot \frac{M}{(E I)_{e f}} \\
& \sigma_{m, c}=0,5 \cdot E_{c} \cdot h_{c} \cdot \frac{M}{(E I)_{e f}} \\
& \sigma_{c}+\sigma_{m, c} \leq f_{c, c}
\end{aligned}
$$

Onde:

$\mathrm{M}=$ momento fletor na seção considerada;

$\sigma_{\mathrm{c}}=$ tensão normal no centroide da área de concreto devido à força normal;

$\sigma_{\mathrm{m}, \mathrm{c}}=$ tensão normal na extremidade da área de concreto devido ao momento; e

$\mathrm{fc}_{, \mathrm{c}}=$ resistência do concreto na compressão.

$\mathrm{Na}$ extremidade inferior da peça mista, a tensão normal de tração na madeira é verificada por meio das seguintes relações (Eq. 11 a 13):

$\sigma_{w}=\gamma_{w} \cdot E_{w} \cdot a_{w} \cdot \frac{M}{(E I)_{e f}}$

Eq. 11

$\sigma_{m, w}=0,5 \cdot E_{w} \cdot h_{w} \cdot \frac{M}{(E l)_{e f}}$

$\sigma_{\mathrm{w}}+\sigma_{\mathrm{m}, \mathrm{w}} \leq \mathrm{f}_{\mathrm{w}, \mathrm{t}}$

Onde:

$\sigma_{\mathrm{w}}=$ tensão normal no centroide da área de madeira devido à força normal;

$\sigma_{\mathrm{m}, \mathrm{c}}=$ tensão normal na extremidade da área de madeira devido ao momento; e

$\mathrm{f}_{\mathrm{w}, \mathrm{t}}=$ resistência da madeira à tração.

A tensão máxima de cisalhamento na linha neutra da viga mista é satisfeita por (Eq. 14):

$$
\tau_{\mathrm{w}, \max }=0,5 \cdot \mathrm{E}_{\mathrm{w}} \cdot \mathrm{h}^{2} \cdot \frac{\mathrm{V}}{(\mathrm{El})_{\mathrm{ef}}} \leq \mathrm{f}_{\mathrm{v}, 0}
$$

Eq. 14 
Onde:

$\mathrm{V}$ = força máxima de cisalhamento na seção considerada; e

$\mathrm{f}_{\mathrm{v}, 0}=$ resistência ao cisalhamento da madeira medida paralelamente às fibras.

A força cisalhante no sistema de conexão é satisfeita por (Eq. 15):

$\mathrm{F}=\gamma_{\mathrm{c}} \cdot \mathrm{E}_{\mathrm{c}} \cdot \mathrm{A}_{\mathrm{c}} \cdot \mathrm{a}_{\mathrm{c}} \cdot \mathrm{s} \cdot \frac{\mathrm{V}}{(\mathrm{El})_{\mathrm{ef}}} \leq \mathrm{R}_{\mathrm{v} 1}$

Onde:

$\mathrm{R}_{\mathrm{v} 1}=$ resistência ao cisalhamento de cada conector $\mathrm{X}$.

Segundo a NBR 6118 (ABNT, 2003), o deslocamento máximo admitido para o concreto é (Eq. 16):

$\mathrm{u}_{\mathrm{q}, \mathrm{lim}} \leq \frac{\mathrm{L}}{500}$

Onde:

$\mathrm{u}_{\mathrm{q}, \mathrm{lim}}=$ limitação do deslocamento para o concreto, medido na direção vertical, no centro do vão, devido à força acidental.

No caso da madeira, o deslocamento máximo segundo a NBR 7190 (ABNT, 1997) é (Eq. 17):

$\mathrm{u}_{\lim } \leq \frac{\mathrm{L}}{200}$

Onde:

$\mathrm{u}_{\mathrm{lim}}=$ limitação do deslocamento para a madeira, medido na direção vertical, no centro do vão, devido à força variável.

\section{Figura 2 - Corpos de prova mistos}

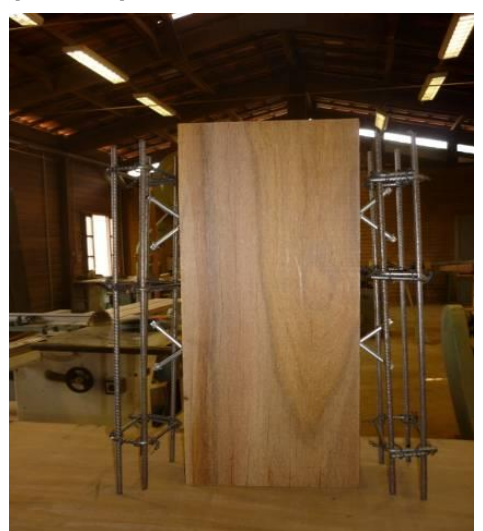

(a) Detalhes dos conectores em X e armadura para o concreto
Vale mencionar também que na última versão de revisão da norma brasileira de madeiras NBR 7190 (ABNT, 2013) a limitação da flecha para uma viga de madeira foi estabelecida em L/300. No entanto, não existem recomendações normativas sobre 0 limite de deslocamentos verticais no meio do vão para vigas mistas de madeira e concreto.

\section{Materiais e métodos}

\section{Corpos de prova mistos}

Inicialmente foram realizados ensaios experimentais de cisalhamento direto em corpos de prova mistos de madeira e concreto para a obtenção da resistência e da rigidez do sistema de conexão do tipo $\mathrm{X}$ utilizado. Para a aplicação de forças nos corpos de prova foi utilizado um pórtico de reação com atuador hidráulico de capacidade $480 \mathrm{kN}$ (48.000 kgf), acionado por controle manual. Foi também utilizado um sistema de aquisição de dados externo, com 20 canais, e três deles foram usados para a recepção dos sinais, sendo um para a célula de carga $(50 \mathrm{kN})$, e os outros dois para os transdutores de deslocamentos. Foi usado um total de dois transdutores de deslocamentos com sensibilidade de $0,001 \mathrm{~mm}$, posicionados em faces opostas do corpo de prova, conforme mostra a Figura 2. A base de medidas dos deslocamentos considerada para os corpos de prova foi igual a $50 \mathrm{~cm}$. Utilizou-se uma célula de carga com capacidade de $50 \mathrm{kN}$ para a medição da força aplicada no corpo de prova durante o ensaio. Um anel acoplado ao pórtico metálico foi também utilizado para o controle da aplicação do carregamento durante o ensaio de cisalhamento direto.

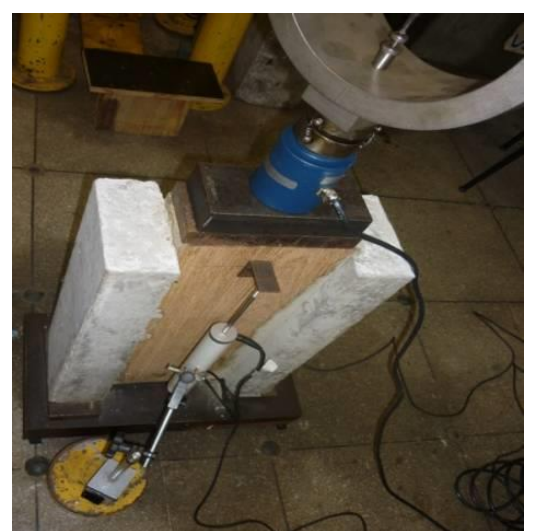

(b) Instrumentação com transdutores de deslocamento

Fonte: Silva (2011). 
As forças nos corpos de prova foram aplicadas em dois ciclos de carregamento, sendo o primeiro com força aplicada até $50 \%$ do valor da força de ruptura (para acomodação do corpo de prova), e o segundo aplicado até a ruptura. Importante informar que foram analisadas duas configurações de corpo de prova (configurações do tipo 1 e 2) em função das diferentes disposições dos conectores $\mathrm{X}$ nos corpos de prova, conforme ilustram as Figuras 3 e 4, apresentadas na sequência. Os corpos de prova do tipo 1 apresentaram dois pares de conectores $\mathrm{X}$ em cada lado do corpo de prova, enquanto os corpos de prova do tipo 2 apresentaram apenas um conector X em cada lado. Foi ensaiado um total de dois corpos de prova do tipo 1 (nomeados por $\mathrm{CP}$ II e CP IV) e dois corpos de prova do tipo 2 (nomeados por CP I e CP III) para a obtenção da resistência e rigidez médias de cada conector $\mathrm{X}$. Os conectores utilizados para compor os corpos de prova foram parafusos do tipo CS100900 com dimensões de $55 \mathrm{~mm} \times 100 \mathrm{~mm}$, com diâmetro de
7,5 mm, conforme mostra a Figura 5. Na composição dos corpos de prova utilizou-se madeira da espécie cumaru com resistência média de compressão de 98,91 $\mathrm{MPa}$ e módulo de elasticidade de 27.796,3 MPa. Foi utilizado também concreto com resistência média de compressão igual a 26,89 MPa e módulo de elasticidade de $21,5 \mathrm{GPa}$. A caracterização da madeira foi feita de acordo com recomendações da norma NBR 7190 (ABNT, 1997), enquanto a caracterização do concreto foi feita com base nas normas NBR 5738 (ABNT, 2003) e NBR 8522 (ABNT, 2008). Na composição das armaduras para o concreto dos corpos de prova foram utilizadas barras de aço CA-50, com diâmetro de $6,3 \mathrm{~mm}$, e estribos de barras de aço CA-60, com diâmetro de $5 \mathrm{~mm}$.

Os detalhes de alguns ensaios de caracterização dos materiais madeira e concreto são apresentados na Figura 6.

Figura 3 - Configuração para o corpo de prova do tipo 1

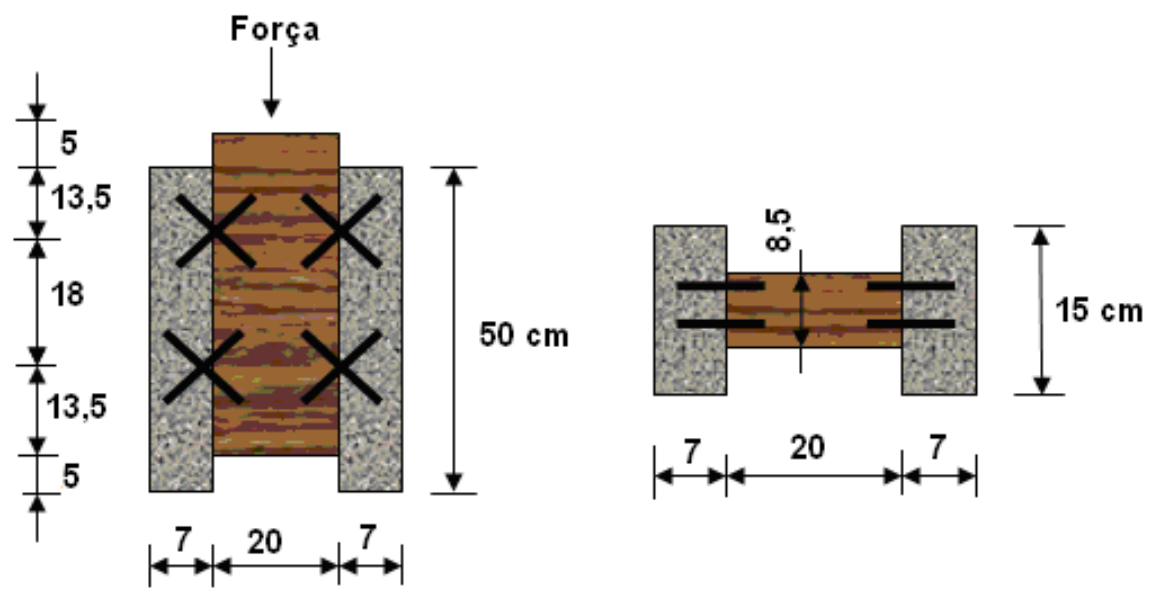

Fonte: Silva (2011).

Figura 4 - Configuração para o corpo de prova do tipo 2

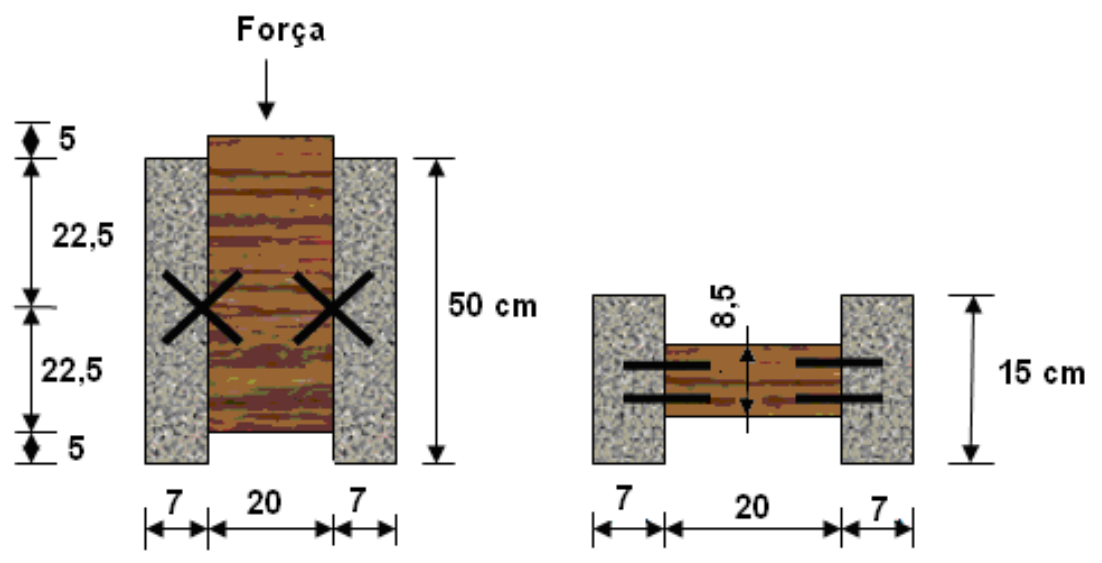

Fonte: Silva (2011). 
Figura 5 - Conectores CS100900 do tipo parafuso para composição dos conectores X

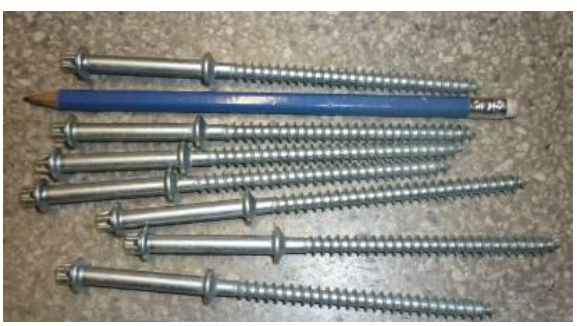

Figura 6 - Caracterização dos materiais

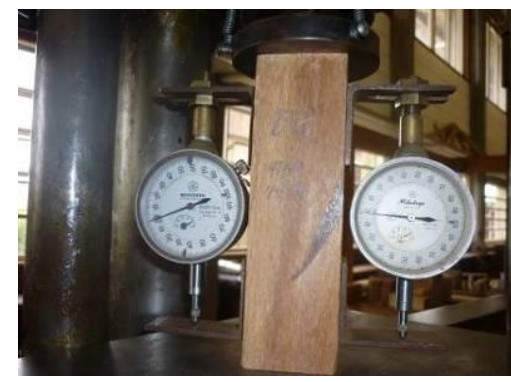

(a) Madeira

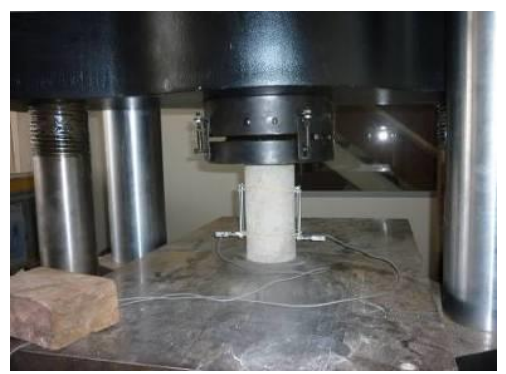

(b) Concreto

Fonte: Silva (2011).

\section{Vigas mistas}

Para as vigas mistas admitiram-se os materiais madeira e concreto com as mesmas propriedades utilizadas para os corpos de prova mistos. Utilizou-se o espaçamento entre conectores recomendado pelo fabricante, igual a $18 \mathrm{~cm}$. O comprimento das vigas foi determinado procurando-se eliminar a parcela de deformação por cisalhamento no valor flecha a partir da consideração da relação entre o comprimento da viga (L) e sua altura (h), maior do que 21 (L / h > 21). As vigas de madeira foram serradas com seção retangular, com dimensões de $8,5 \mathrm{~cm} \times 12$ $\mathrm{cm}$ x $370 \mathrm{~cm}$, sendo a mesa de concreto de dimensões $7 \mathrm{~cm}$ x $35 \mathrm{~cm}$, e a distância entre apoios de $350 \mathrm{~cm}$. Foi utilizado um total de 19 pares de conectores em $\mathrm{X}$, distribuídos ao longo do comprimento da viga mista, conforme ilustra a Figura 7.

Os detalhes dos conectores em $\mathrm{X}$, assim como os das armaduras utilizadas para o concreto e concretagem das vigas, são apresentados na Figura 8. Nas armaduras do concreto foram utilizadas barras de aço CA-50 com 6,3 mm de diâmetro, posicionadas ao longo do comprimento (vigas) e 20 estribos de aço CA-60 com diâmetro de 5 mm, espaçados em $18 \mathrm{~cm}$.
As armaduras utilizadas nas mesas das vigas mistas representaram armaduras mínimas com relação ao volume de concreto utilizado e foram utilizadas com o objetivo de reduzir as fissurações no concreto durante as realizações dos ensaios de flexão. Os ensaios das vigas e dos corpos de prova foram realizados no Laboratório de Madeira e Estrutura de Madeira - LaMEM da USP, em São Carlos, SP.

$\mathrm{Na}$ realização dos ensaios de flexão das vigas mistas também foram utilizados os mesmos equipamentos utilizados para os corpos de prova, ou seja, o pórtico de reação, o sistema de aquisição de dados externos e, ainda, três transdutores de deslocamentos com percurso máximo de $50 \mathrm{~mm}$, para a leitura da medida dos deslocamentos. Dois dos transdutores de deslocamentos foram posicionados nas extremidades da viga mista para verificação do deslocamento nos apoios, e o terceiro transdutor, centralizado na parte de baixo (centro do vão) da viga mista. A Figura 9 mostra os detalhes da instrumentação e ensaio das vigas mistas. Os carregamentos foram aplicados no centro do vão das vigas até a ruptura delas, para a avaliação da resistência e da rigidez do sistema de conexão em X utilizado. 
Figura 7 - Configuração utilizada para as vigas mistas

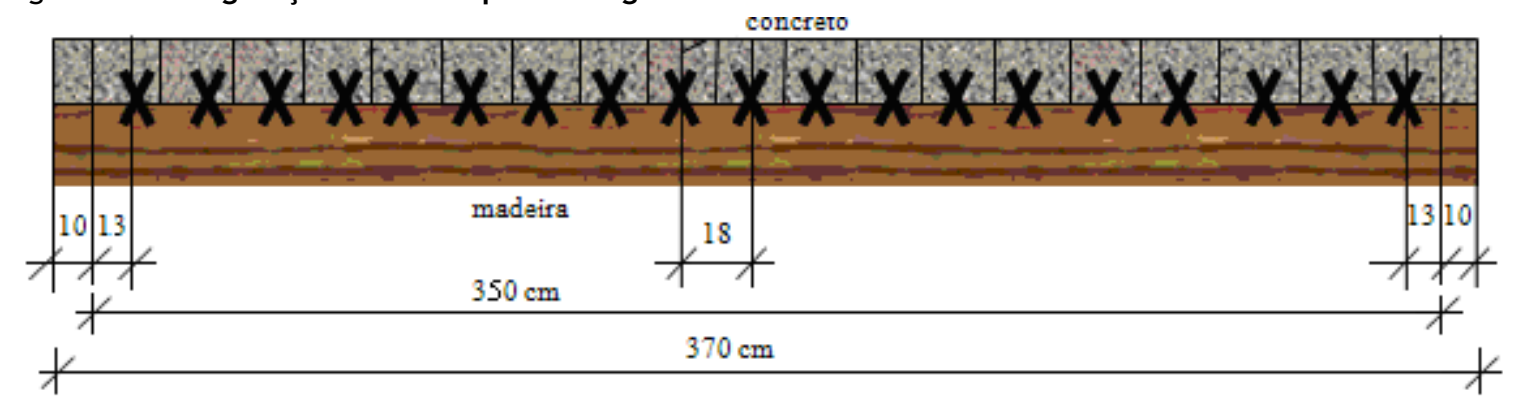

Fonte: Silva (2011).

Figura 8 - Detalhes da confecção das vigas mistas

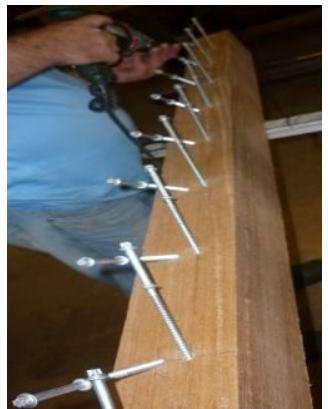

(a) Conectores de cisalhamento em X

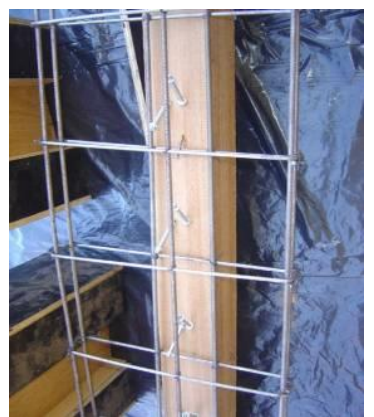

(b) Armadura para o concreto

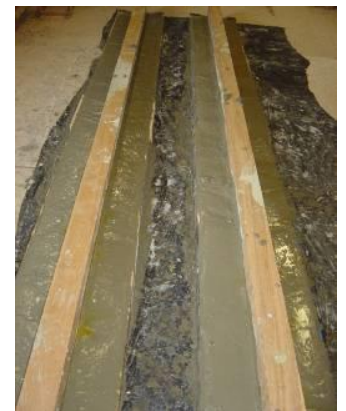

(c) Concretagem das vigas mistas

Fonte: Silva (2011).

Figura 9 - Detalhes da instrumentação e do ensaio de flexão das vigas mistas

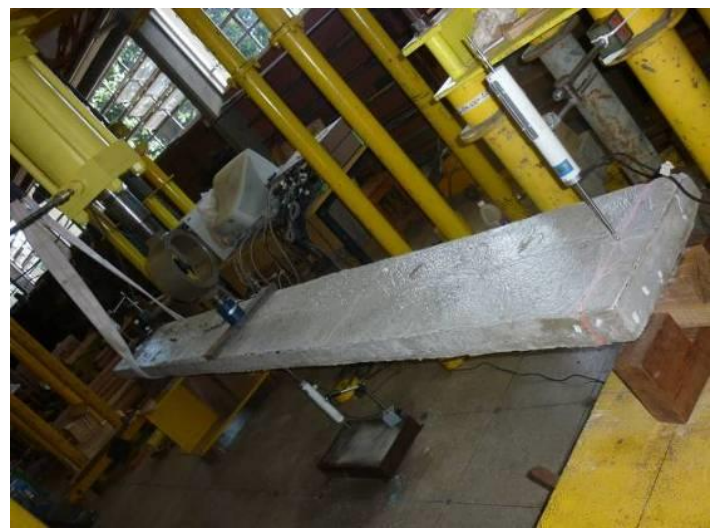

Fonte: Silva (2011).

\section{Resultados e discussão \\ Corpos de prova mistos}

Os ensaios de cisalhamento foram feitos a partir da média de dois corpos de prova (CP I e CP III), com um par de conectores $\mathrm{X}$, e a partir de dois corpos de prova (CP II e CP IV), com dois pares de conectores X. A partir dos resultados dos corpos de prova com um par de conectores $\mathrm{X}$ (configuração do tipo 2) observou-se que a força média última de ruptura foi de aproximadamente
43 kN. Neste caso, a resistência média, convencionada a deformação em $2 \%$, foi de 32,50 $\mathrm{kN}$. A Figura 10 mostra a curva força versus deformação para um dos corpos de prova com configuração do tipo 2 .

O módulo de deslizamento $\mathrm{K}$ (força/deslocamento) foi obtido com base nos valores $10 \%$ e $40 \%$ da resistência, convencionada a deformação em $2 \%$, conforme recomendações da norma brasileira de madeiras NBR 7190 (ABNT, 1997). O valor do módulo de deslizamento médio $(\mathrm{K})$, neste caso, foi 
igual a $19,94 \mathrm{kN} / \mathrm{mm}$ para o par de conectores X. A Figura 11 mostra a curva força versus deslocamento para o corpo de prova com configuração do tipo 2.

Para o corpo de prova com dois pares de conectores (configuração do tipo 1), a força média de ruptura obtida foi de $64 \mathrm{kN}$. A resistência média, convencionada a deformação em $2 \%$, neste caso, foi de $60 \mathrm{kN}$, conforme mostra a Figura 12. O módulo de deslizamento médio foi de 28,88 $\mathrm{kN} / \mathrm{mm}$ para dois pares de conectores, com base nas informações da Figura 13.

Figura 10 - Curva força $\mathrm{x}$ deformação para corpos de prova com configuração do tipo 2

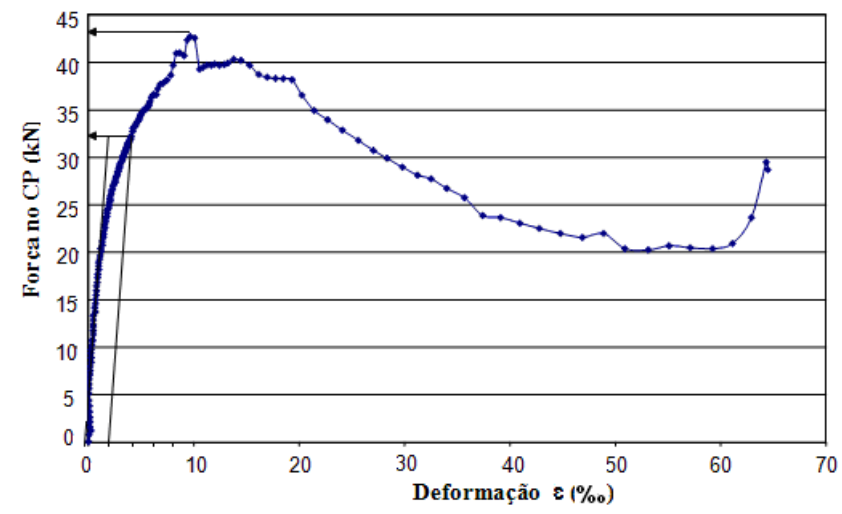

Figura 11 - Curva força $\mathrm{x}$ deslocamento para corpos de prova com configuração do tipo 2
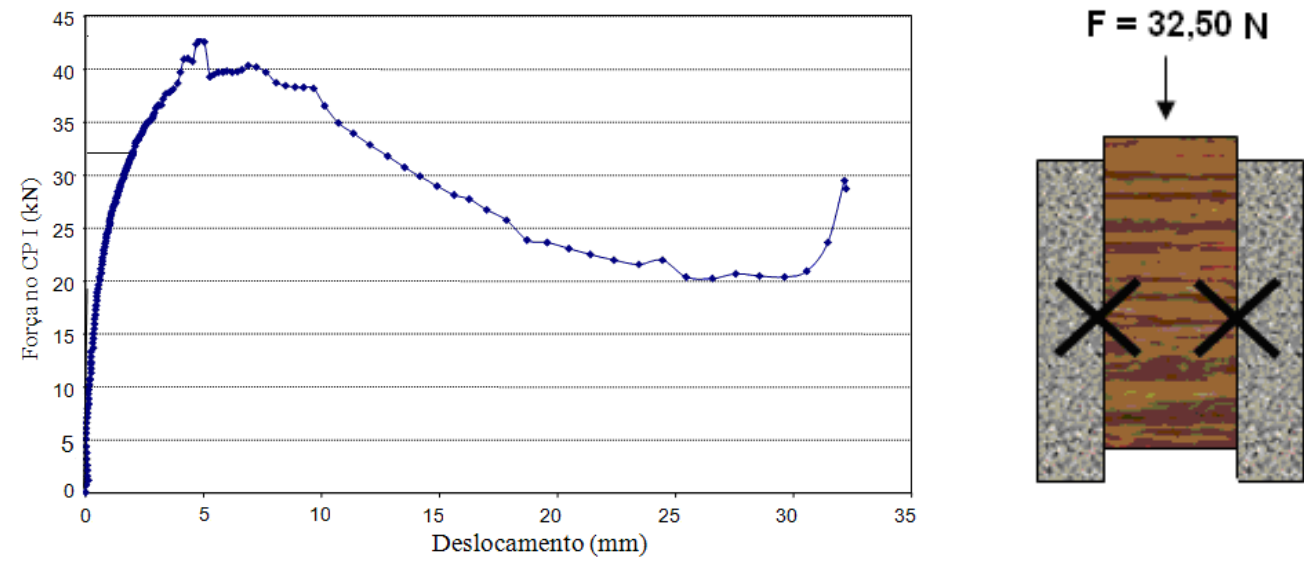

Figura 12 - Curva força $x$ deformação para corpos de prova com configuração do tipo 1

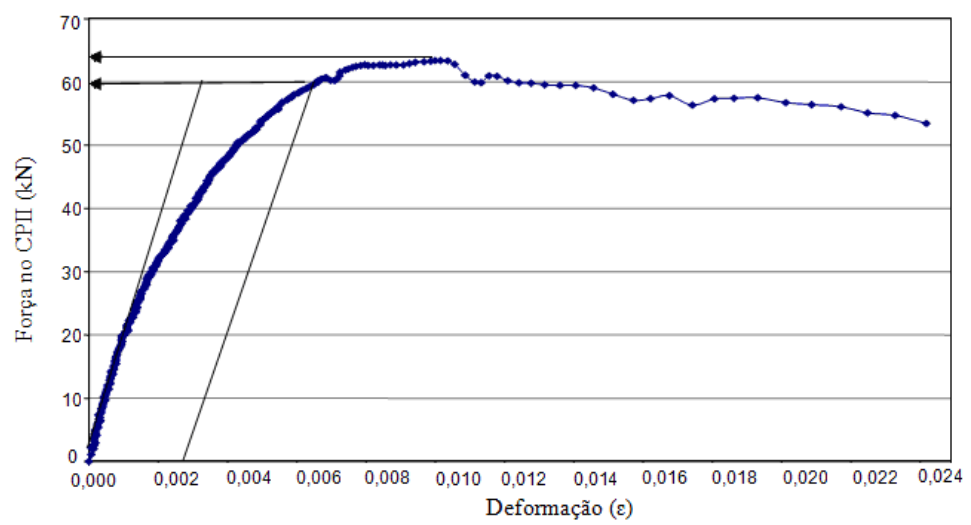

36 Molina, J. C.; Silva, M. A. de A. A. da; Vasconcelos, R. P. de 
Figura 13 - Curva força $x$ deslocamento para corpos de prova com configuração do tipo 1
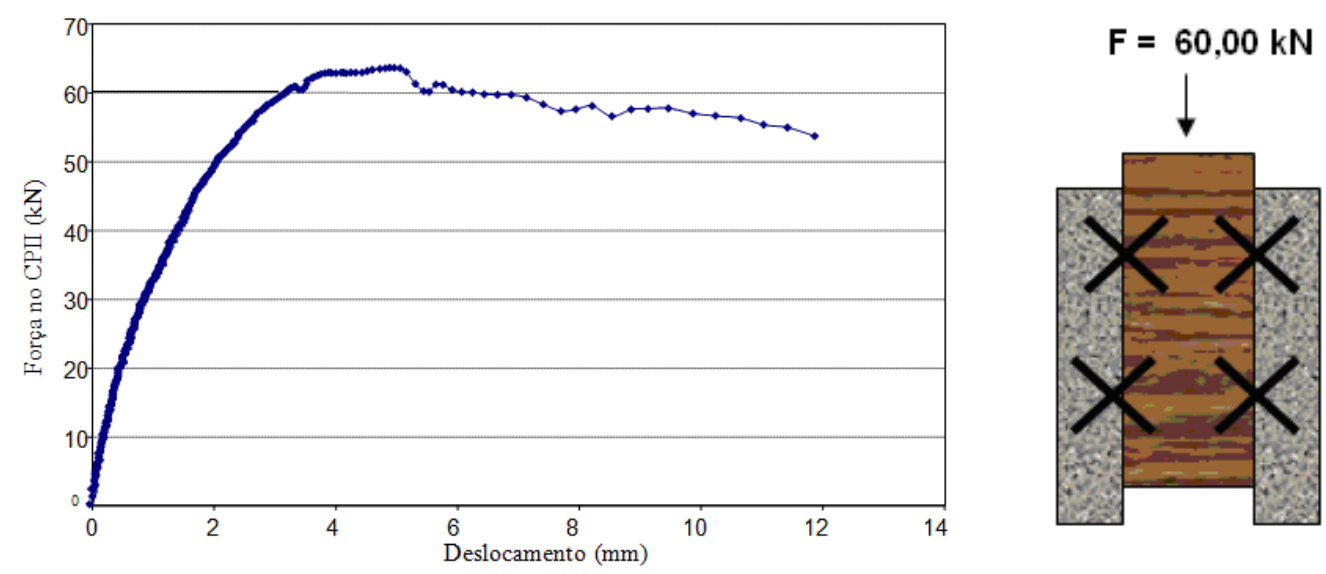

O valor médio obtido para o módulo de deslizamento foi de $13,38 \mathrm{kN} / \mathrm{mm}$ para cada conector $\mathrm{X}$, e a resistência média igual a $15,62 \mathrm{kN}$. Vale observar ainda que, para os corpos de prova com configuração do tipo 2 , a resistência de cada conector foi de $16,25 \mathrm{kN}$, e para os corpos de prova com configuração do tipo 1 a resistência de cada conector foi de $15,00 \mathrm{kN}$. Nos corpos de prova com configuração do tipo 1 , observou-se que os conectores superiores resistiram primeiramente ao nível de força aplicada, e os conectores inferiores passaram a trabalhar quando os conectores superiores já haviam apresentado deformações.

\section{Vigas mistas}

Foram ensaiadas duas vigas mistas (nomeadas por VM I e VM II), com a mesma configuração, para a determinação os valores de força última e rigidez EI. O valor médio de rigidez experimental $(\mathrm{EI})_{\exp }$ foi comparado com o valor analítico $(\mathrm{EI})_{\mathrm{ef}}$, obtido através do modelo de Mohler. Para as vigas analisadas, os valores das forças últimas de ruptura foram iguais a $51 \mathrm{kN}$ no caso da viga VM I e a 48 $\mathrm{kN}$ no caso da viga VM II. Os valores experimentais das flechas foram de $90 \mathrm{~mm}$ para VM I e de $95 \mathrm{~mm}$ para VM II. A força aplicada foi também estimada graficamente para a flecha L/300, o que corresponde a aproximadamente 1,20 $\mathrm{cm}$ (distância entre apoios de $350 \mathrm{~cm}$ ). Neste caso, a força que provocou a referida flecha foi de $11 \mathrm{kN}$ para a viga VM I e de aproximadamente $15 \mathrm{kN}$ para a viga VM II, como mostra a Figura 14.

Os valores experimentais de rigidez $(\mathrm{EI})_{\exp }$ foram calculados a partir das flechas (u) e forças (F) obtidas para cada viga (Eq. 18).

$E I_{\exp }=\frac{F \cdot L^{3}}{48 . u}$

Eq. 18
Sendo assim, o valor médio de rigidez experimental (EI) calculado para forças de serviço com aproximadamente $50 \%$ dos valores de ruptura, obtidos nos ensaios de flexão, foi de aproximadamente $9045178 \mathrm{kN} . \mathrm{cm}^{2}$ e de 5917418 $\mathrm{kN} . \mathrm{cm}^{2}$ para forças últimas de ruptura. Observouse assim que o valor experimental médio obtido para a rigidez $(\mathrm{EI})_{\text {exp }}$, para carregamentos de serviço com $50 \%$ do valor de ruptura, apresentou uma diferença de aproximadamente $34,5 \%$ com relação ao valor da rigidez $(\mathrm{EI})_{\exp }$ obtido para os estados limites últimos. Do gráfico da Figura 14(a), observa-se que a viga mista VM I apresentou um comportamento aproximadamente linear até a força aplicada de $45 \mathrm{kN}$, com comportamento levemente não linear a partir desse ponto até a ruptura. Esse comportamento é devido à aplicação de ciclos de força para a acomodação dela. Por outro lado, na Figura 14(b), o gráfico para a viga VM II apresentou dois intervalos distintos, e o primeiro trecho, entre o início do ensaio até aproximadamente a força aplicada de 20 $\mathrm{kN}$, apresentou comportamento relativamente linear; já a partir do valor de força de $20 \mathrm{kN}$, a curva mudou de inclinação, tendo apresentado comportamento não linear, mantendo-se então linear até a ruptura da viga. Esse comportamento da curva indica que a viga não teve, inicialmente, uma acomodação satisfatória em função da não utilização de ciclos de carga-descarga para acomodação do sistema. Nesse sentido, durante o ensaio de vigas mistas é necessário que sejam realizados pelo menos dois ciclos de carregamento (carga-descarga), sendo um com metade da força última de ruptura obtida por ensaio em viga gêmea, para acomodação do sistema, e o segundo ciclo com valor de força aplicado até a ruptura. $\mathrm{Na}$ análise visual após os ensaios de cisalhamento dos corpos de provas e das vigas foi observado que ocorreram algumas falhas nas regiões dos 
conectores, devido ao embutimento do conector de aço na madeira, enquanto os danos no concreto foram de pequena magnitude. Nos conectores comprimidos foi observada a tendência de formação de rótulas plásticas. A Figura 15 mostra os modos de falha na região dos conectores após a ruptura do sistema. Observou-se que os danos nos conectores dos corpos de prova foram mais acentuados que nos conectores das vigas mistas. Esse comportamento pode ser explicado em função de se ter cisalhamento puro na conexão, no caso do ensaio de cisalhamento dos corpos de prova, enquanto nos conectores das vigas houve melhor

Figura 14 - Curvas força $x$ flecha

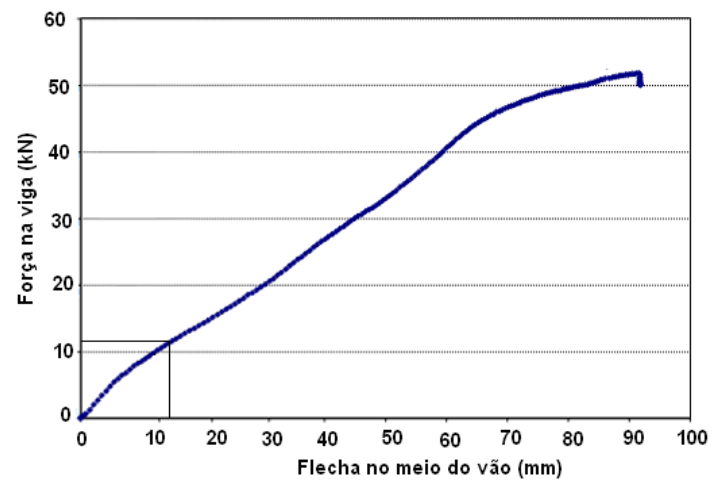

(a)VM I $(\mathrm{F}=11 \mathrm{kN}$ para $\mathrm{u}=1,20 \mathrm{~mm})$ distribuição dos esforços internos, com solicitações conjuntas de tração, compressão e cisalhamento.

De uma maneira geral, nos conectores de aço dispostos em $\mathrm{X}$ os mecanismos de deformações dos elementos tracionados $\left(\mathrm{F}_{\mathrm{t}}\right)$ provocam embutimento na madeira, enquanto nos elementos comprimidos $\left(\mathrm{F}_{\mathrm{c}}\right)$ formam rótulas plásticas, limitando sua resistência, além do embutimento na madeira. Portanto, para o conector "inclinado", a solicitação axial de tração é a que melhor se aproxima das condições de serviço do conector, como mostra a Figura 16.

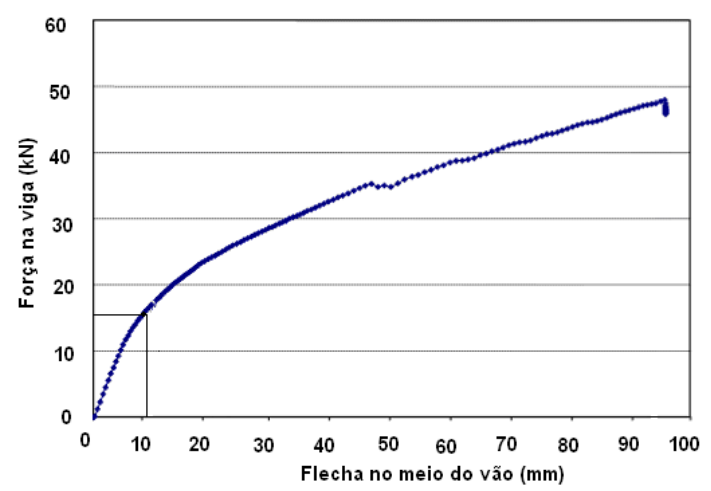

(b) VM II ( $\mathrm{F}=15 \mathrm{kN}$ para $\mathrm{u}=1,20 \mathrm{~mm})$

Figura 15 - Modos de falha e deformação dos conectores: tendência de arrancamento dos conectores tracionados e formação de rótulas plásticas nos conectores comprimidos

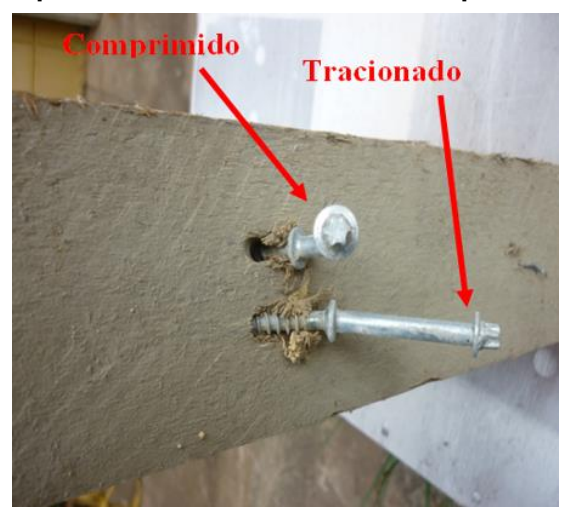

Figura 16 - Deformações nos conectores inclinados

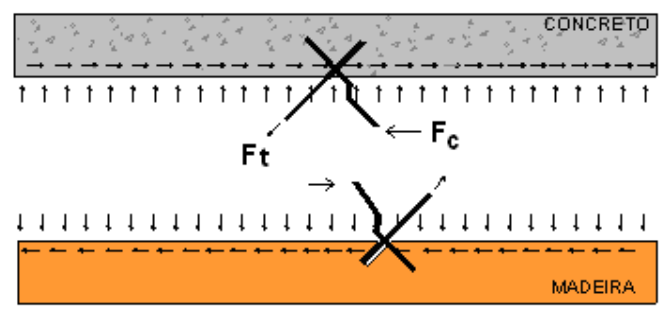

Fonte: adaptado de Pigozzo (2004). 
Os danos na conexão ocorrem devido ao fato de existir concentração de tensões nas regiões dos conectores. Assim, enquanto toda a viga trabalha dentro do regime linear, as regiões dos conectores podem atingir a resistência última dos materiais envolvidos na ligação mista.

\section{Estimativa da ruptura dos materiais: Modelo de Mohler}

A partir do modelo de Mohler a ruptura estimada da viga ocorreu primeiramente na mesa de concreto para uma força média de $29,89 \mathrm{kN}$, seguida do cisalhamento dos conectores $\mathrm{X}$ para uma força média $37,46 \mathrm{kN}$. Os demais modos de ruptura na viga estimados foram a tração na fibra inferior da madeira, com força média de $57,62 \mathrm{kN}$, e então por cisalhamento na LN, para uma força média de 525,51 kN. Por outro lado, o valor médio de rigidez teórico $(\mathrm{EI})_{\mathrm{ef}}$ obtido a partir do modelo de Mohler foi igual a $9285600 \mathrm{kN} . \mathrm{cm}^{2}$ para forças com $50 \%$ do valor de ruptura e de 8695921 $\mathrm{kN} . \mathrm{cm}^{2}$ para valores de força última aplicada.

Para forças de serviço a relação entre o valor experimental de rigidez (EI) $)_{\exp }$ e aquele obtido pelo modelo de Mohler (EI) foi de aproximadamente $97,3 \%$, enquanto para forças últimas aplicadas a mesma relação de rigidez foi de $68,01 \%$, o que indica que o modelo de Mohler apresentou boa aproximação dos valores de rigidez (EI) para força de serviço com $50 \%$ do valor de ruptura. Por outro lado, para valores últimos de força aplicada nas vigas, o modelo de Mohler apresentou diferenças consideráveis de até $32 \%$ no valor da rigidez $(\mathrm{EI})_{\mathrm{ef}}$ com relação ao valor obtido experimentalmente $(\mathrm{EI})_{\exp }$. Para carregamento de serviço com valores de até $50 \%$ dos valores das força de ruptura, as diferenças (teóricas e experimentais) nos valores de rigidez EI foram de aproximadamente $2,6 \%$.

Com base no exposto, vale mencionar também que o modelo de Mohler considera a rigidez da conexão de cisalhamento, e este permite avaliar tanto as tensões (ELU) como os deslocamentos (ELS). No entanto, como o objetivo principal deste estudo foi realizar uma análise preliminar da rigidez (EI) a partir da comparação do modelo de Mohler e ensaios experimentais, não foi realizada a instrumentação das vigas mistas com extensômetros para a avaliação das tensões e deformações. Neste caso, as respostas de rigidez EI foram avaliadas experimentalmente a partir das flechas máximas fornecidas em função dos carregamentos de serviço e último aplicados. Também não foi desenvolvido nenhum modelo de elementos finitos para a análise do sistema de conexão considerado, já que o interesse da pesquisa também não foi a avaliação por simulação numérica do nível de tensões nas regiões dos conectores de cisalhamento, nem na viga como um todo.

\section{Conclusões}

O modelo analítico de Mohler, o qual está apresentado no Eurocode 5 (EUROPEAN..., 2001), pode ser utilizado na avaliação do comportamento de rigidez na flexão de vigas mistas de madeira e concreto com seção transversal $\mathrm{T}$.

A resposta estrutural de rigidez $(\mathrm{EI})_{\mathrm{ef}}$ fornecida pelo modelo teórico de Mohler corresponde a uma análise elástica. Nesse sentido, na avaliação da rigidez (EI), na flexão de vigas mistas de madeira e concreto, o modelo garante boa aproximação para os resultados experimentais de rigidez (EI) quando são forças aplicadas nas vigas com até $50 \%$ do valor de ruptura obtido em ensaios experimentais. Nesse caso a diferença entre os valores teóricos e experimentais de rigidez (EI) são de aproximadamente 2,6\%. Por outro lado, para valores últimos de forças aplicadas nas vigas mistas, os valores teóricos fornecidos pelo modelo de Mohler e aqueles obtidos experimentalmente para a rigidez (EI) divergem em até $32 \%$.

Os valores de rigidez EI obtidos a partir do modelo de Mohler foram menos conservadores que os mesmos valores obtidos experimentalmente.

$\mathrm{Na}$ análise da rigidez (EI) de vigas mistas a partir de ensaios de flexão é importante de sejam aplicados pelo menos dois ciclos de carregamento com carga e descarga para acomodação do sistema, sendo o último ciclo com força aplicada até a ruptura.

Os conectores de cisalhamento do tipo CS100900 utilizados em $X$ nas vigas mistas de madeira e concreto analisadas apresentaram bom comportamento no que se refere à resistência e à rigidez. Os valores médios de resistência e de rigidez para esse tipo de conector $\mathrm{X}$ foram de $13,38 \mathrm{kN} / \mathrm{mm}$ e $15,62 \mathrm{kN}$ respectivamente.

No que se refere aos mecanismos de deformação da conexão formada por parafusos do tipo CS100900 dispostos em X, pode-se dizer que os parafusos tracionados tendem a provocar embutimentos na madeira, enquanto os parafusos comprimidos tendem a formar rótulas plásticas, além do embutimento na madeira.

Como sugestão para trabalhos futuros, recomendase o valor limite da flecha igual a L/300 para ensaios não destrutivos de flexão estática em vigas mistas de madeira e concreto, sendo L o tamanho 
do vão entre apoios. Esse limite está de acordo com as recomendações da última versão de revisão da norma NBR 7190 (ABNT, 2013) para vigas de madeira submetidas à flexão.

\section{Refrências}

ASSOCIAÇÃO BRASILEIRA DE NORMAS TÉCNICAS. NBR 7190: projeto de estrutura de madeira: procedimento. Rio de Janeiro, 1997.

ASSOCIAÇÃO BRASILEIRA DE NORMAS TÉCNICAS. NBR 7190: $2^{\circ} \mathrm{PN}$ revisão: projeto de estrutura de madeira: procedimento. Rio de Janeiro, 2013.

\section{ASSOCIAÇÃO BRASILEIRA DE NORMAS} TÉCNICAS. NBR 5738: concreto: procedimento para moldagem e cura de corpos de prova: procedimento. Rio de Janeiro, 2003.

\section{ASSOCIAÇÃO BRASILEIRA DE NORMAS} TÉCNICAS. NBR 8522: determinação dos módulos estáticos de elasticidade e de deformação e da curva tensão-deformação: procedimento. Rio de Janeiro, 2008.

\section{ASSOCIAÇÃO BRASILEIRA DE NORMAS} TÉCNICAS. NBR 6118: projeto de estrutura de concreto: procedimento. Rio de Janeiro, 2003.

BRANCO, J. M., CRUZ, P. J. Ligações Mistas Madeira-Betão Leve. In: SIMPÓSIO EPUSP SOBRE ESTRUTURAS DE CONCRETO, 5., São Paulo, 2003. Anais... São Paulo: EPUSP, 2003.

\section{EUROPEAN COMMITTEE FOR STANDARTIZATION. EUROCODE 5: ENV} 193 1-1: design of timber structures: general rules and rules and buildings.Brussels, 2001.

MOLINA, J. C. Análise do Comportamento Dinâmico da Ligação Formada Por Barras de Aço Coladas Para Tabuleiros Mistos de Madeira e Concreto Para Pontes. São Carlos,2008. 240 f. Tese (Doutorado em Engenharia Civil) - Escola de engenharia, Universidade de São Paulo, São Carlos, 2008.

PIGOZZO, J. C. Estudo e Aplicações de Barras de Aço Coladas Como Conectores em Lajes Mistas de Madeira e Concreto Para Tabuleiros de Pontes. São Carlos, 2004. 343 f. Tese (Doutorado em Engenharia de Estruturas) - Escola de Engenharia, Universidade de São Paulo, São Carlos, 2004.

SILVA, M. A. A. A. Aplicação de Conectores Metálicos em Pontes de Concreto e Madeira para Estradas Vicinais no Estado do Amazonas. Manaus, 2012. 113 f. Dissertação (Mestrado em Engenharia Civil) - Escola de Engenharia, Universidade Federal do Amazonas, Manaus, 2012.

\section{Agradecimentos}

Agradecemos à Capes, pelo apoio financeiro, sem o qual esta pesquisa não poderia ter sido realizada.

\footnotetext{
Julio Cesar Molina

Universidade Estadual Paulista Júlio de Mesquita Filho | Rua Geraldo Alckimin, 519, Nossa Senhora de Fátima | Itapeva - SP - Brasil | CEP 13560-970 | Tel.: (15) 3524-9100 | E-mail: molina@itapeva.unesp.br
}

Marcia Almeida de Amaral Arcos da Silva

Departamento de Construção, Faculdade de Tecnologia | Universidade Federal do Amazonas | Av. Gal. Rodrigo Octávio Jordão Ramos, 3000, Aleixo | Manaus - AM - Brasil | CEP 69077-000 | Tel.: (92) 3305-4410 | E-mail: marcinha.arcos@hotmail.com

Raimundo Pereira de Vasconcelos

Departamento de Construção, Faculdade de Tecnologia | Universidade Federal do Amazonas | Tel.: (92) 3305-4410

E-mail: vasconcelos@ufam.edu.br

Revista Ambiente Construído

Associação Nacional de Tecnologia do Ambiente Construído

Av. Osvaldo Aranha, $99-3^{\circ}$ andar, Centro

Porto Alegre - RS - Brasil CEP $90035-190$

Telefone: +55 (51) 3308-4084

Fax: +55 (51) 3308-4054

www.seer.ufrgs.br/ambienteconstruido

E-mail: ambienteconstruido@ufrgs.br

40 Molina, J. C.; Silva, M. A. de A. A. da; Vasconcelos, R. P. de 